\title{
Factores de conversión del pepino del mar
}

\author{
Conversion factors of sea cucumber
}

\author{
Brianda Montenegro Hurtado ${ }^{1}$ \\ Donald Williams Smith ${ }^{2}$
}

\section{Resumen}

El pepino del mar (Holotúridas), se ha convertido en un nuevo producto de exportación de Nicaragua, cobrando auge comercial cada vez mayor y han sido sujeto a la explotación comercial que promueven una fuente significativa de empleo e ingresos a comunidades costeras del Caribe Norte de Nicaragua. El estudio es cuantitativo con carácter descriptivo de corte transversal con una duración de 3 meses. Se estudiaron los factores de conversión de tres especies de Holotúridas realizados por el Instituto Nicaragüense de Pesca y Acuicultura en la ciudad de Bilwi, de marzo a mayo del 2017; para ello, la muestra poblacional fue de 95 individuos vivos de las cuales 31 individuos de Holothuria floridana, 64 individuos de Isostichopus badionotus y 69 individuos de Holothuria mexicana obteniendo un peso total de 51.72 libras de ejemplares frescos con vísceras y el porcentaje de reducción de peso debido a los diferentes procesos que lleva el pepino del mar para llegar hasta su conversión final (peso seco), fue de la siguiente; para la primera especie fue de $91.96 \%$ de pérdida de su peso inicial, para la segunda fue de $96.11 \%$ de pérdida de su peso inicial y 91.30\% para la tercera especie.

Palabras clave: Factores de conversión; Holuturidas; muestra poblacional; proceso.

\section{Abstract}

The sea cucumber (Holoturidas), has become a new export product from Nicaragua, charging increasing commercial boom and have been subject to commercial exploitation that promote a significant source of employment and income to coastal communities from the North Caribbean of Nicaragua. The study is quantitative with a descriptive character of cross sectional scope with a duration of 3 months. The conversion factors of three Holoturidas species carried out by the Nicaraguan Institute of Fisheries and Aquaculture in Bilwi city, from March to May 2017, were studied; for this, the population sample was 95 living individuals of which 31 individuals of Holothuria floridana, 64 individuals of Isostichopus badionotus and 69 individuals of Mexican Holothuria obtaining a total weight of 51.72 pounds of fresh specimens with viscera and the percentage of weight reduction due to the different processes that the sea cucumber takes to reach its final conversion (dry weight), it was as follows; for the first species it was 91.96\% of its initial weight loss, for the second it was $96.11 \%$ of its initial weight loss and $91.30 \%$ for the third species.

Keywords: Conservation Factors; Holuturidas; population sample; process.

\section{Introducción}

La pesca del pepino de mar ha diversificado el aprovechamiento de los recursos pesqueros tradicionales (caracol, langosta, escamas, crustáceos) en la Costa Caribe de Nicaragua, siendo prioridad para los pescadores artesanales en temporada de veda de la langosta y caracol; esta actividad, se realiza mayormente de forma artesanal por medio de embarcaciones menores como panga, Ponkin y velero, lo cual a través del buceo a pulmón, tanques o compresor de aire extraen este recurso. En el 2008, la comercialización de este

\footnotetext{
1 Licenciada en Biología, Instituto Nicaragüense de Pesca y Acuicultura, Técnica de pesca, e-mail: brianda_mha@yahoo.es

2 Licenciado en biología marina, Técnico de IREMADES, Tutor de investigación; ORCID http://orcid.org/0000-0002-1931-577X; e-mail; ronaldw1981@yahoo.com
}

Recibido: 08/08/2019 - Aprobado: 30/08/2019 
recurso se ha incrementado en la Región Autónoma Costa Caribe Norte, principalmente extraen las especies Holothuria mexicana, (pepino molongo), Isostichopus badionotus (pepino café) y Holothuria floridana (conocido como pepino carajo).

Según, estudios realizados por la UNAN- León y UNAM- México, en las aguas del caribe nicaragüense se encuentran un total de 10 especies de Holuturidas, de las cuales hasta el momento 6 especies tienen importancia comercial Isostichopus badionotus (Café), Holothuria floridana (Pikachu), Holothuria mexicana (Molongo), Astichopus multifidus (Toalla), petate y pepino de piedra.

Con referencia a lo anterior, el INPESCA a través de la Dirección de Investigaciones Pesqueras ha realizado estudios enfocados a las diferentes especies de pepino de mar de importancia comercial que se capturan en las regiones del mar Caribe, designándose como lugar de investigación la ciudad de Bilwi, Puerto Cabezas, donde existe mayormente la pesca de pepino del mar y esta investigación fue realizado entre el mes de marzo, mayo y julio del 2017.

Debido a la falta de información de conversión de pepino del mar (mermación del producto), fue necesario determinar el porcentaje de pérdida de pesos en sus diferentes presentaciones (fresco, cocido, seco), a fin de estimar las unidades en número de individuos y peso que se está comercializando y la cantidad que se extrae de su medio natural para cumplir la cuota de exportación como peso seco.

\section{Revisión de literatura}

\section{Generalidades de la familia Holothuroidea}

Las Holoturias tienen forma alargada, la boca y el ano no se encuentran en las zonas más extremas del cuerpo, si no en posición ligeramente ventral debido a que tienen un lado del cuerpo en contacto con el sustrato, y no la región anal o bucal como los otros equinodermos. Esto ha conducido a que se desarrolle una simetría bilateral secundaria, en la que se distingue una zona ventral, en contacto con el sustrato, llamada trivio, y una zona dorsal, llamada vivio (Edwards E. Ruppert et al. 1996).

Edwards et. al (1996). afirma; que los pepinos del mar tienen colores que van del negro al castaño rojizo, pasando por el verde oscuro y miden entre dos centímetros y dos metros de longitud y la locomoción es por medio de varios pequeños pies y sus tentáculos que sirven para recoger partículas comestibles que llevan a su boca. Algunas especies de pepinos de mar expelen hilos blancos pegajosos para enredar o distraer a potenciales depredadores e inclusive expulsan sus órganos interiores cuando son perturbados y extraordinariamente, esto no mata al pepino de mar, que simplemente regenera sus órganos.

Están ampliamente distribuidos en todos los océanos, desde las zonas intermareales hasta las profundidades oceánicas y son muy comunes en los océanos Índico y Pacífico sur occidental, y en los trópicos se observa fácilmente en el fondo arenoso del mar, entre corales o piedras.

Los pepinos de mar son un recurso importante para las comunidades costeras y los ecosistemas marinos. La función principal de las holotúridas es la bioturbación de sedimentos y el reciclaje de material orgánico. (Birkeland 1988).

\section{Importancia ecológica de los pepinos de mar}

Los pepinos de mar tienen una importante función en los ecosistemas del fondo del mar, en los que son capaces de producir substanciales cambios físicos, químicos y físico-químicos por la ingestión intensiva y selectiva de la capa superficial del sedimento y por el enriquecimiento ambiental que producen sus deposiciones (Francour, 1998). 
Al igual que las lombrices, los pepinos de mar consumen y pican materia sedimentaria y orgánica en compuestos más finos, invirtiendo las capas superiores de los fondos marinos, lo que permite que el oxígeno penetre en el sedimento. Esto impide la acumulación de materia orgánica que puede ayudar a controlar los patógenos (Francour, 1998).

Los pepinos de mar son equinodermos que ayudan a que la plataforma insular se mantenga limpia y alejada del peligro de toxicidad que los desechos orgánicos acumulan y que atentan con la vida y el normal desarrollo de los corales; además, reciclan las partículas orgánicas y enriquecen los substratos (Hudson et al. 2004).

Además de su función en el ecosistema el pepino de mar se ha convertido en un nuevo producto de exportación de Nicaragua, cobrando un auge cada vez mayor, muchas especies de holotúridos han sido sujetos a la explotación comercial que promueven una fuente significativa de empleo e ingresos a comunidades costeras y son altamente valiosos en el mercado asiático.

\section{Importancia económica de los pepinos de mar}

La pesquería del pepino de mar comenzó hace más de mil años en el lejano oriente. En el siglo XIX esta pesquería se expandió, con China como el principal importador mundial de pepinos de mar durante el presente siglo se abrieron al comercio Internacional también los mercados de Japón, Corea, Singapur y Taiwán (Conand et al. 1988), independientemente de la razón de su uso es innegable que el pepino de mar cuenta con una gran demanda y se mueven millones de dólares por la importación y exportación de algunas de sus especies (Herrera D. 2001).

Según la FAO, los pepinos del mar se comercializan en forma seca, a menudo llamado "beche de mar o trepang" y el comercio internacional aumentó dramáticamente en los años ochenta, alcanzando un volumen global de 10,000 toneladas anuales de pepino del mar seco.

Desde la perspectiva socioeconómica como ecológica, las sostenibilidades a largo plazo de la pesquería del pepino de mar son de gran importancia para las familias costeñas, desde tiempos inmemorables, la pesca ha sido una de las principales fuentes de alimento de las comunidades costeras, una fuente de identidad cultural, y un proveedor de empleo y beneficios económicos para quienes se dedican a esta actividad.

La conservación y el manejo de los pepinos de mar son de extrema importancia por cuanto este animal cumple un rol importante en el ecosistema marino y son una fuente importante de ingresos para muchas comunidades costeras del Caribe de Nicaragua.

Desde el 2008, la comercialización de este recurso se ha incrementado, En la Región Autónoma Costa Caribe Norte, principalmente extraen las especies Holothuria mexicana, (pepino molongo), Isostichopus badionotus (pepino café) y Holothuria floridana ( pepino carajo); por lo, que se hace necesario contar con los factores de conversión de cada uno de ellos para conocer los porcentajes de pérdida de pesos en sus diferentes estados (fresco, cocido, seco), a fin de estimar las unidades en número de individuos y peso que se está comercializando.

\section{Materiales y métodos}

El presente trabajo investigativo se elaboró en el tema de factores de conversión de pepino del mar realizado por medio de un estudio cuantitativo con carácter descriptivo de corte transversal con una duración de 3 meses. El universo de la muestra poblacional fue de 164 individuos vivos de las cuales; 31 individuos era de la especie Holothuria floridana, 64 individuos de Isostichopus badionotus y 69 individuos de Holothuria mexicana

Las muestras fueron brindadas por parte de los pescadores y centros de acopios de la ciudad de Bilwi, Puerto Cabeza, la cual se utilizaron los siguientes procedimientos: 
1) Recibida las muestras vivas (individuos con vísceras) en los centros de acopios, procedió a pesar y la medición biométrica de cada individuo, posteriormente se eviscero por individuo y pesó nuevamente la muestra total (peso fresco sin vísceras) en libras, registrándose los datos en los formatos establecidos para dicho estudio.

2) Una vez eviscerados, se procedieron a cocer dentro de un recipiente de aluminio con agua dulce en un fogón conectado mediante una manguera a un tanque de gas propano a una temperatura de 60-70 grados promedio, moviéndolos constantemente con un cucharon de madera por 20 minutos, para realizar el primer cocido, este proceso se aplicó a las dos especies, se tomaron datos de peso, se dejaron enfriar para ubicarlos en un recipiente de plástico con abundante sal, durante 12 horas, para la especie Isostichopus badionotus; en el caso del Holoturia floridana se procedió a resguardar en el cuarto frio, para el día siguiente exponerlo al sol e iniciar a obtener el valor por día del peso seco; la muestra brindada por el acopio Vlazar Costa Caribe S.A (Isostichopus badionotus), fue secada en horno artesanal (cuarto recubierto de láminas de zinc lisas, con ventiladores y fogata.

3) Para la especie Holothuria mexicana una vez eviscerados, se procedieron a cocer dentro de un recipiente de aluminio con agua dulce en un fogón conectado mediante una manguera a un tanque de gas propano a una temperatura de 70 grados promedio, moviéndolos constantemente con un cucharon de madera por 15 minutos hasta alcanzar la temperatura máxima el recipiente para realizar el primer cocido.

4) Posteriormente se tomaron datos de peso, seguidamente se ubicaron en salmuera en un recipiente de plástico con agua de mar (3 galones) y sal granulada (5 libras), durante 14 horas. Para el día siguiente exponerlo al sol e iniciar a obtener el valor por día del peso seco.

\section{Herramientas utilizadas para el muestreo:}

Balanza digital Recipiente de aluminio

Cinta métrica $(\mathrm{cm})$

Balde plástico

\section{Resultados y discusión}

Las especies utilizadas para este estudio de factores de conversión son las siguientes; Isostichopus badionotus, Holothuria floridana y Holothuria mexicana. En el siguiente cuadro muestra el número de individuos utilizados por especies y la mermación del producto atraves de los diferentes procesos utilizados:

Cuadro No. 1. Merma de dos especies de pepino del mar

\begin{tabular}{|l|l|l|l|l|}
\hline \multicolumn{1}{|c|}{ Nombre científico } & Holothuria floridana & \multicolumn{3}{c|}{ Isostichopus badionotus } \\
\hline \multicolumn{1}{|c|}{ Proceso } & \multicolumn{1}{|c|}{ Secado al sol } & \multicolumn{1}{c|}{ Secado al sol } & \multicolumn{1}{c|}{ Secado al horno } & Total Promedio \\
\hline Número de individuos & 31 & 21 & 43 & 64 \\
\hline Peso Fresco con Vísceras (lb) & 20.98 & 25.90 & 60.18 & 86.08 \\
\hline Peso Fresco sin Vísceras (lb) & 9.10 & 15.30 & 26.45 & 41.75 \\
\hline Reducción (\%) & 56.63 & 40.93 & 56.05 & 51.50 \\
\hline Peso primer cocido (lb) & 4.45 & 4.10 & 11.24 & 15.34 \\
\hline Reducción (\%) & 51.10 & 73.20 & 57.50 & 63.26 \\
\hline Peso después de salado (lb) & 4.15 & 3.50 & 10.36 & 13.86 \\
\hline Reducción (\%) & 6.74 & 14.63 & 7.83 & 9.65 \\
\hline
\end{tabular}




\begin{tabular}{|l|l|l|l|l|}
\hline \multicolumn{1}{|c|}{ Nombre científico } & Holothuria floridana & \multicolumn{3}{c|}{ Isostichopus badionotus } \\
\hline \multicolumn{1}{|c|}{ Proceso } & \multicolumn{1}{|c|}{ Secado al sol } & \multicolumn{1}{c|}{ Secado al sol } & \multicolumn{1}{c|}{ Secado al horno } & Total Promedio \\
\hline Peso después de 12 horas de congelado (lb) & 4.41 & 3.78 & & 3.776 \\
\hline Peso después de 2da cocida (lb) & & 3.47 & 7.93 & 11.40 \\
\hline Reducción (\%) & 6.31 & 8.21 & 23.46 & 17.78 \\
\hline Peso Seco (lb) & 1.6874 & 1.0075 & 1.76 & 2.7675 \\
\hline Reducción (\%) & 61.75 & 70.93 & 77.81 & 75.72 \\
\hline Reducción de peso fresco con vísceras a seco (\%) & 91.96 & 96.11 & 97.08 & 96.78 \\
\hline
\end{tabular}

En el cuadro anterior, presentan los datos de la Merma del pepino del mar desde peso fresco con vísceras hasta peso seco. Para, el cálculo de los factores de conversión de las dos especies antes descrito, se utilizó una muestra total de 95 individuos de las cuales 64 corresponde a Isostichopus badionotus y 31 a Holothuria floridana. Los resultados determinan que la mayor pérdida de peso corresponde a la especie I. badionotus con una reducción del $96.78 \%$, la especie H. floridana reduce hasta un $91.96 \%$ de su peso inicial (fresco con vísceras).

La talla mínima de la especie Isostichopus badionotus fue de $17.6 \mathrm{~cm}$ y la talla máxima de $36.7 \mathrm{~cm}$, para la especie Holothuria floridana la talla mínima fue de 12.5 y la máxima de $28 \mathrm{~cm}$.

\section{Factores de conversión de Holothuria mexicana realizados por INPESCA y CAMPRESA}

En los estudios realizados durante los años 2006 al 2008 por el Instituto Nicaragüense de la Pesca y Acuicultura (INPESCA) y la empresa Mariscos y Pescados S.A. (CAMPRESA), se calcularon los factores de conversión para la especie Holothuria mexicana, con una muestra de 50 individuos que pesaron 70 libras de peso fresco con vísceras y un peso final de 4.10 libras (peso seco).

Cuadro No. 2. Factores de conversión del Holothuria mexicana

\begin{tabular}{|c|c|c|c|}
\hline Convertir & a & en & Multiplicar por \\
\hline \multirow{3}{*}{1 individuo } & Peso fresco & \multirow{12}{*}{ LIBRAS } & 1.400 \\
\hline & Peso precocido & & 0.320 \\
\hline & Peso seco & & 0.082 \\
\hline \multirow{3}{*}{1 libra fresco } & No. individuos & & 0.714 \\
\hline & Peso precocido & & 0.228 \\
\hline & Peso seco & & 0.058 \\
\hline \multirow{3}{*}{1 libra precocido } & No. individuos & & 3.125 \\
\hline & Peso fresco & & 4.375 \\
\hline & Peso seco & & 0.256 \\
\hline \multirow{3}{*}{1 libra seco } & No. individuos & & 12.195 \\
\hline & Peso fresco & & 17.073 \\
\hline & Peso precocido & & 3.902 \\
\hline
\end{tabular}

En el cuadro No. 2, muestran los datos que se obtuvieron de la especie Holothuria floridana que fue de 31 individuos, para un peso fresco con vísceras de 20.98 libras, 4.45 libras de precocido y un peso final seco de 1.687 libras. Para obtener, el peso por individuo se dividió el peso obtenido en cada proceso por el número de individuos, dando como resultado que un individuo pesa 0.6768 libras fresco con vísceras, 0.1435 libras precocido y 0.0544 seco con estos datos se trabajaron para obtener los factores de conversión que se presentan en el cuadro siguiente. 
Cuadro No. 3. Peso de la muestra de Holothuria floridana y peso por individuo

\begin{tabular}{|l|l|l|l|l|l|l|c|}
\hline \multicolumn{1}{|c|}{ Localidad } & No. Individuos & \multicolumn{1}{|c|}{$\begin{array}{c}\text { Fresco } \\
\text { (libras) }\end{array}$} & Fresco/NI & $\begin{array}{c}\text { Precocido } \\
\text { (libras) }\end{array}$ & \multicolumn{1}{|c|}{ Precocido/NI } & $\begin{array}{c}\text { Seco } \\
\text { (libras) }\end{array}$ & seco/NI \\
\hline Puerto Cabezas & 31 & 20.98 & 0.6768 & 4.45 & 0.1435 & 1.6874 & 0.0544 \\
\hline
\end{tabular}

NI: número de individuos

Cuadro No. 4. Factores de Conversión del Holothuria floridana

\begin{tabular}{|c|c|c|c|}
\hline Convertir & $a$ & en & Multiplicar por \\
\hline \multirow{3}{*}{1 individuo } & Peso fresco & \multirow{12}{*}{ LIBRAS } & 0.6768 \\
\hline & Peso Precocido & & 0.1435 \\
\hline & Peso seco & & 0.0544 \\
\hline \multirow{3}{*}{1 libra fresco } & No. individuos & & 1.4776 \\
\hline & Peso Precocido & & 0.2121 \\
\hline & Peso seco & & 0.0804 \\
\hline \multirow{3}{*}{1 libra Precocido } & No. individuos & & 6.9663 \\
\hline & Peso fresco & & 4.7146 \\
\hline & Peso seco & & 0.3792 \\
\hline \multirow{3}{*}{1 libra seco } & No. individuos & & 18.3715 \\
\hline & Peso fresco & & 12.4333 \\
\hline & Peso Precocido & & 2.6372 \\
\hline
\end{tabular}

De acuerdo, a los factores calculados para Holothuria floridana, nos Indica que 1 libra de pepino seco para ser exportado equivale a 12.4333 libras de pepino fresco con vísceras, 2.6372 libras de pepino precocido y 18 individuos. En tanto, 1 libra de pepino fresco con vísceras equivale a 0.0804 libras de pepino seco listo para ser exportado, 0.2121 libras de pepino precocido y 1.5 individuos.

\section{Isostichopus badionotus secados al sol}

En el siguiente cuadro indica una muestra de 21 individuos pesados para un total de 25.9 libras (fresco con vísceras) y cada individuo tiene un peso promedio de $1.23 \mathrm{lb}$, una vez cocida la muestra tiene un peso de 3.47 libras y un peso promedio de 0.1650 libras por individuo y finalmente la muestra seca peso 1.0075 libras, para un peso por individuo de 0.048 libras.

Cuadro No. 5. Peso por individuo de Isostichopus badionotus secado al sol

\begin{tabular}{|c|c|c|c|c|c|c|c|}
\hline \multirow{2}{*}{ Localidad } & \multirow{2}{*}{ No. Individuos } & \multirow{2}{*}{ Fresco (Ib) } & \multirow{2}{*}{ Fresco/NI } & Precocido & \multirow{2}{*}{ Precocido/NI } & \multirow{2}{*}{ Seco (lb) } & \multirow{2}{*}{ seco/N } \\
\hline & & & & (lb) & & & \\
\hline Puerto Cabezas & 21 & 25.9 & 1.2333 & 3.47 & 0.1650 & 1.0075 & 0.048 \\
\hline
\end{tabular}

NI: Número de Individuo

Estos datos, se calcularon los factores de conversión, que se presentan en el cuadro No.5 
Cuadro No. 6. Factores de conversión Isostichopus badionotus (secado al sol)

\begin{tabular}{|c|c|c|c|}
\hline Convertir & a & en & Multiplicar por \\
\hline \multirow{3}{*}{1 individuo } & Peso fresco & \multirow{12}{*}{ LIBRAS } & 1.2333 \\
\hline & Peso precocido Precocido & & 0.1650 \\
\hline & Peso seco & & 0.0480 \\
\hline \multirow{3}{*}{1 libra fresco } & No. individuos & & 0.8108 \\
\hline & Peso Precocido & & 0.1338 \\
\hline & Peso seco & & 0.0389 \\
\hline \multirow{3}{*}{1 libra Precocido } & No. individuos & & 6.0589 \\
\hline & Peso fresco & & 7.4726 \\
\hline & Peso seco & & 0.2907 \\
\hline \multirow{3}{*}{1 libra seco } & No. individuos & & 20.8437 \\
\hline & Peso fresco & & 25.7072 \\
\hline & Peso Precocido & & 3.4402 \\
\hline
\end{tabular}

De acuerdo, a los factores calculados para Isostichopus badionotus secada al sol, nos indica que 1 libra de pepino seco para ser exportado equivale a 25.7072 libras de pepino fresco con vísceras, a 3.4402 libras de pepino precocido y a 20.8437 individuos.

En tanto 1 libra de pepino fresco con vísceras equivalen a 0.0389 libras de pepino seco listo para ser exportado, a 0.0389 libras de pepino precocido y a 0.8108 individuos.

\section{Peso por individuos secado en horno de Isostichopus badionotus}

En cuanto, al factor de conversión de Isostichopus badionotus secado al sol la muestra fue de 43 individuos con un peso total fresco con vísceras de 60.18 libras, precocido 7.93 libras de y 1.76 libras de peso seco. En cuanto, a los pesos por individuos fueron de 1.3995 peso fresco con vísceras, 0.1844 precocido y 0.0409 peso seco.

Cuadro No. 7. Peso por individuos secado en horno de Isostichopus badionotus

\begin{tabular}{|c|c|c|c|c|c|c|c|}
\hline \multirow{2}{*}{ Localidad } & \multirow{2}{*}{ No. Individuos } & \multirow{2}{*}{ Fresco (Lb) } & \multirow{2}{*}{ Fresco/NI } & Precocido & \multirow{2}{*}{ Precocido/NI } & \multirow{2}{*}{ Seco (Lb) } & \multirow{2}{*}{ seco/N } \\
\hline & & & & (Lb) & & & \\
\hline Puerto Cabezas & 43 & 60.18 & 1.3995 & 7.93 & 0.1844 & 1.76 & 0.0409 \\
\hline
\end{tabular}

NI: Número de Individuo

Con estos datos se calcularon los factores de conversión, que se presentan en la siguiente tabla. 
Cuadro No. 8. Factores de conversión de Isostichopus badionotus secado en horno

\begin{tabular}{|c|c|c|c|}
\hline Convertir & a & en & Multiplicar por \\
\hline \multirow{3}{*}{1 individuo } & Peso fresco & \multirow{9}{*}{ Libras } & 1.3995 \\
\hline & Peso precocido & & 0.1844 \\
\hline & Peso seco & & 0.0409 \\
\hline \multirow{3}{*}{1 libra fresco } & No. individuos & & 0.7145 \\
\hline & Peso precocido & & 0.1318 \\
\hline & Peso seco & & 0.0292 \\
\hline \multirow{3}{*}{1 libra Precocido } & No. individuos & & 5.4224 \\
\hline & Peso fresco & & 7.5889 \\
\hline & Peso seco & & 0.2219 \\
\hline \multirow{3}{*}{1 libra seco } & No. individuos & \multirow{3}{*}{ Libras } & 24.4318 \\
\hline & Peso fresco & & 34.1932 \\
\hline & Peso precocido & & 4.5057 \\
\hline
\end{tabular}

De acuerdo a los factores calculados para Isostichopus badionotus secada en horno, nos Indica que 1 libras de pepino seco para ser exportado equivalen a 34.1932 libras de pepino fresco con vísceras, 4.5057 libras de pepino precocido y 24.4318 individuos. En tanto 1 libra de pepino fresco con vísceras equivalen a 0.0292 libras de pepino seco listo para ser exportado, a 0.1318 libras de pepino precocido y 0.7145 individuos.

Como indica en la siguiente tabla, se presentan datos de pérdida de peso a partir del peso inicial (peso fresco con víscera) hasta peso final (peso seco) de la muestra obtenida en los cayos Miskitus.

Cuadro No. 9. Muestreo de pérdida de peso fresco a peso seco en los cayos Miskitus

\begin{tabular}{|l|l|}
\hline \multicolumn{2}{|c|}{ Secado al sol } \\
\hline Nombre local & Molongo \\
\hline Número de Individuos & 69 \\
\hline Peso Fresco con Vísceras (libras) & 51.72 \\
\hline Peso Fresco sin Vísceras (libras) & 29 \\
\hline Reducción (\%) & 43.93 \\
\hline Peso Primer cocido (libras) & 14 \\
\hline Reducción (\%) & 51.72 \\
\hline Segundo peso Cocido (libras) & 8.32 \\
\hline Peso Seco (libras) & 4.5 \\
\hline Reducción (\%) & 45.91 \\
\hline Reducción de peso fresco con vísceras a seco (\%) & 91.3 \\
\hline
\end{tabular}

El porcentaje de reducción del peso fresco con vísceras a fresco sin víscera para la especie Holothuria mexicana, el promedio es de $43.93 \%$ y después del primer cocido sin vísceras es de $51.72 \%$, posteriormente la reducción del proceso de peso seco y primer cocido es de $50.86 \%$ para una pérdida total de $86.7 \%$ de su peso corporal.

Para la toma de los datos de pérdida y porcentajes de reducción, se trabajó con una muestra de 69 individuos frescos, datos obtenidos de la especie Holothuria mexicana en los cayos Miskitus en el año 2017. 
Cuadro No. 10. Peso de la muestra de Holothuria mexicana y peso por individuo

\begin{tabular}{|l|l|l|l|l|l|l|}
\hline \multicolumn{1}{|c|}{$\mathrm{N}^{\circ}$ individuos } & $\begin{array}{c}\text { Peso fresco } \\
\text { libras }\end{array}$ & $\begin{array}{c}\text { Lb/individuo } \\
\text { fresco }\end{array}$ & Peso seco & Lb/individuo seco & $\begin{array}{c}\text { peso precocido } \\
\text { libras }\end{array}$ & $\begin{array}{c}\text { Lb/individuo } \\
\text { precocido }\end{array}$ \\
\hline 69 & 51.72 & 0.7496 & 4.5 & 0.0652 & 14 & 0.2029 \\
\hline
\end{tabular}

En el cuadro $\mathrm{N}^{\circ} .10$, indican los datos que se obtuvieron de la especie Holothuria mexicana que fue 69 individuos, para un peso fresco con vísceras de 51.72 libras, 14 libras de precocido y un peso final seco de 4.5o libras y para poder obtener el peso por individuo, se dividió el peso obtenido en cada proceso por el número de individuos.

\begin{tabular}{|c|c|c|c|}
\hline Convertir & a & en & Multiplicar por \\
\hline \multirow{3}{*}{1 individuo } & Peso fresco & \multirow{12}{*}{ LIBRAS } & 0.7496 \\
\hline & Peso precocido & & 0.2029 \\
\hline & Peso seco & & 0.0652 \\
\hline \multirow{3}{*}{1 libra fresco } & No. individuos & & 1.3340 \\
\hline & Peso precocido & & 0.2707 \\
\hline & Peso seco & & 0.0870 \\
\hline \multirow{3}{*}{1 libra precocido } & No. individuos & & 4.9285 \\
\hline & Peso fresco & & 3.6944 \\
\hline & Peso seco & & 0.3214 \\
\hline \multirow{3}{*}{1 libra seco } & No. individuos & & 15.3333 \\
\hline & Peso fresco & & 11.4939 \\
\hline & Peso precocido & & 3.1111 \\
\hline
\end{tabular}

De acuerdo, a los cálculos anteriores realizados para Holothuria mexicana nos indica que 1 libra de pepino seco para ser exportado equivale a 11.4939 libras de pepino fresco con vísceras, a 3.1111 libras de pepino precocido y a 15.3333 individuos. En tanto 1 libra de pepino fresco con vísceras equivalen a 0.0870 libras de pepino seco listo para ser exportado, a 0.2707 libras de pepino precocido y a 1.3340 individuos.

\section{Conclusiones}

El estudio sobre el factor de conversión de tres especies de pepino de mar fue realizado con una muestra de 95 individuos vivos de las cuales 31 era de la especie Holothuria floridana, 64 de Isostichopus badionotus y 69 individuos vivos de Holothuria mexicana obteniéndose un peso de la muestra de 51.72 libras (fresco con vísceras).

Como resultado de lo anterior, se logró calcular el porcentaje de reducción de peso de las tres especies de pepino del mar llevado a cabo por los diferentes procesos que conlleva hasta el producto final (pepino seco), dando como resultado para la especie Holothuria floridana una pérdida de 91.96\% de su peso inicial 
(peso fresco), para la especie Isostichopus badionotus fue de $96.11 \%$ y $97.08 \%$, para la muestra secada en horno artesanal.

Mientras que el rendimiento para Holothuria floridana fue de $8.04 \%$ y para el Isostichopus badionotus fueron de $3.89 \%$ secada al sol y $2.92 \%$ secada en horno. En cuanto, a la especie Holothuria mexicana (molongo), muestra una pérdida total de su peso de $91.30 \%$ en relación a su peso inicial (peso fresco con víscera) y con un rendimiento de $8.70 \%$.

Por los resultados, obtenidos de las tres especies de pepino del mar comerciales en el Caribe Norte es necesario realizar estudios sobre la biología y población existentes en el mar caribe con el fin de proteger y conservar el recurso pepino de mar muy importante para los ecosistemas marinos y poblaciones costeras.

Asimismo, es necesario promover diferentes acciones enfocadas para un aprovechamiento sostenible del recurso, tales acciones pueden ser; respetar las vedas establecidas, cumplir y respetar las normas y decretos de regulación establecidas por INPESCA, las tallas de captura y comercialización del producto, establecer cotas de captura por especie y temporada de pesca.

\section{Agradecimiento}

Esta publicación obtuvo el financiamiento del Proyecto Ecosistemas Costeros, auspiciado por: Unión Europea, Dka Austria, Cooperación Austriaca para el Desarrollo y Horizontzooo.

\section{Lista de referencias}

Birkeland, C. (1998). The influence of echinoderms on coral-reef communities. Echinoderm Studies, 3:1-79.

Conand, C. (1990). The fishery resources of Pacific island countries. Part 2: Holothurians. FAO Fisheries Technical Paper. No. 272.2, Roma. 143 pp.

Conand, C. y M. Byrne. (1994). Recent evolution of the world fisheries for sea cucumbers. Mar. Fish. Rev., 55(4): $1-13$.

Edwards E. Ruppert et al. (1996). Zoología de invertebrados. Sexta edición México Pág. 974-984.

INPESCA (2017). Muestreos de pepino de mar, para el cálculo de factores de conversión en la región Autónoma de la Costa Caribe Norte (RACCN). 14 pág.

INPESCA (2017). Muestreo de pepino de mar de la especie Holothuria mexicana Molongo), Para el recálculo de factores de conversión. 